\title{
Estado de la nutrición en niños impedidos
}

\author{
Dra. Sylvia Pino R. ${ }^{1}$; Dr. Nelsun Vargas C.. \\ Nutritional status in handicapped children
}

\begin{abstract}
In a sample made up by $30 \%$ (260 cases $\}$ of children under 15 years of age admitcd along 1985 to an institution for the rehabilitation of handicapped children (Instituto de Rehabilitación Infantil) nutritional status was evaluated with WHO reference normal values for weight for age (W/A) and weight for height (W/H). Prevalence of undemutrition was $71 \%$ by $W / A$ and $44.2 \%$ by W/H. These figutes are higher than those for general population. The frequency of advanced undernutrition was $11.5 \%(30$ cases) by W/A and $2.3 \%(6$ cases) by $W / H$. Prevalence was also higher among children between 6 to 14 years of age reaching $80.8 \%$ by W/A and $49.4 \%$ by W/H.

(Key words: Neurological handicaps, undernutrition).
\end{abstract}

La obtención y mantención de un buen estado nutritivo en niños impedidos enfrenta, como es obvio, dificultades mayores que las habituales. La parálisis cerebral, los defectos físicos agregados, las deficiencias en la comunicatión, pueden constituirse en obstáculos serios que, en ocasiones, se suman al sentimiento de frustración. impotencia o desaliento que la limitación puede ocasionar en padres o cuidadores.

Por estas razones, parece lógico suponer que la prevalencia y severidad de la desnutrición sean mayores en estos niños, al comparar con coetá. neos $\sin$ estas deficiencias. En un estudio anterior ${ }^{1}$ pudimos comprobar está aseveración en niños ingrezados a) Instituto de Rehabilitación Infantil (IRI). Sin embargo, por limitaciones del método $y$ diseño empleados en esa ocasión se resolvió realizar el estudio presente, cuyo propósito fue practicar una medición más precisa del

1. Instituto de Rehabilitación Infantil (IRI). Departamento de Pediatría. División Cjencias Médicas Occidente. Facultad de Medicina. Universidad de Chile.

2. Departamento de Salud Pública. Departamento de Pediatría. División Ciencías Médicas Occidente. Facultad de Medicina, Uniyersidad de Chile. problema, para proponer medidas de control, especificas para estos niños.

Los objetivos del trabajo fueron establecer la prevalencia de desnutrición (D) total y por grados según edad, sexo y grupo etario, según la relación pesoledad $(\mathrm{P} / \mathrm{E})$ utilizando los valores normales de la Organización Mundial de la Salud $^{2}$. Igual se hizo con la relación peso talla (P/T) y establecer la distribución de la $\mathrm{D}$, según diagnóstico de ingreso al instituto $y$, en especial, en pacientes con parálisis cerebral. Sc tomó este grupo especifico por ser el diagnóstico más frecuente de ingreso a la institución.

\section{MATERIAL Y METODO}

Se tomó al azar, 260 niñus ingr esados entre marzo $y$ diciembre de 1985. $30 \%$ de los ingresos totales en ese período). Se ubtuvo, así, una muestra comparable en distribucion por sexo, al total de ingresos y con edades que flutuaron entre 6 meses y 14 años.

Se excluyeron los niños ampurados, pues la carencia de extremidades altera la determinación y comparabilidad del peso.

Se determinó el cstado nutritivo utilizando liss tablas de la O.M.S. y calculando, en cada njīo, la raciación $P / \mathbf{E}$ y $P$ T .

Fintre los estudiados hubo 156 niños con parálisis cerebrat, patologia en la que la dependencia alimentaria 
es muy alta; siendo, por lo tanto, de inter és medir más exactamente los grados de $\mathrm{D}$.

Para los efectos de clasificación se consideró obesos a los niños cuyas deteminaciones se ubicaron sobre dos desviaciones tipos del valor central; sobrepeso, a los situados entre $+2 y+1$ DT: eutrofia, entre $-1 y+1$ DT; desnutrido I, entre J y 2 D'T: desmutrido II, cлtre 2 y -3 DT: desnutrido lll a aquellos bajo 3 DT.

El peso se obtuvo pesando los niñus desnudos. Los lactantes en balanza "Scca". con vensibilidad de 20 g $y$ los pre-escolares y escolates en balanra "Hispana", de precision, con sensibilidad de $100 \mathrm{~g}$. I.a talla se deter minó en formia habitual. con curtalión o por segmertos. en alquellos nínos cuyo impedimentis hacía imposible medislos en extensión.

\section{RESULTADOS}

La Tabla I presenta el análisis de comparabilidad. por edad y sexo, ertre los 260 nitios estudiados y los 795 ingresados en igual perio. do. La proporción de pre-escolarcs csta sobresrepresentada en los niños estudiados, mientras que ocurrió lo inverso con los escolares. No hay diferencia sjgnificativa en la proporción de lactantes.

El estado nutritivo, por sexo, según la relación PiE se presenta en la Tabla 2 . Un $71 \%$ de los niños tuvo algún grado de desnutrición, siendo el grado I el más frecuente. No hubo variaciones de importancia en la comparación por sexo.

Lú Tabla 3 presenta el estado nutritivo por edad según $P / E$. Sc observa un aumento gradual en la prevalencia de D desde los lactantes $(64,7 \%)$ a los precscolares $(67,1 \%)$ y escolares $(80,8 \%)$. Este ineremento se realiza en base de los grados tnás avancados de D (II y III).

La Tabla 4 presenta la prevalencia de $D$, por edad según la relación $P / T$. Cumo sc ha probado en estudios anteriores ${ }^{3}{ }^{4}$ esta metodología (relación $\mathrm{P} / \mathrm{T}$ ), da cifras significativamente inferiores de prevalencia. El aumento de la $\mathrm{D}$ en los grupos etarios avanzados, que se observó con la relación $\mathrm{P}$ if, sólo se detecta en escolares, cn base de la $\mathrm{D}_{2}$.

El estudiar la prevalencia de D. en los 160 niños con parálisis cerebral, la relación $P / T$, mos-

Tabla 1

Comparación por sexo y edad de niños ingresados y estudiados I.R.l. Marzo a diciembre 1986. Distribución porcentual

\begin{tabular}{|c|c|c|c|c|c|c|}
\hline \multirow{2}{*}{$\begin{array}{l}\text { Edad } \\
\text { (Años) }\end{array}$} & \multicolumn{3}{|c|}{ Ingresados } & \multicolumn{3}{|c|}{ Estudiados } \\
\hline & $\begin{array}{c}\text { Hombres } \\
(n=429)\end{array}$ & $\begin{array}{l}\text { Mujwes } \\
(n=366)\end{array}$ & $\underset{(n=795)}{\text { Total }}$ & $\begin{array}{l}\text { Hombres } \\
(n=131)\end{array}$ & $\begin{array}{l}\text { Mujeres } \\
(n=129)\end{array}$ & $\begin{array}{c}\text { Total } \\
(n=260)\end{array}$ \\
\hline-2 & $11.7^{\circ}$ & 15,3 & 13,3 & 14,5 & 11,6 & 13,0 \\
\hline $2-5$ & 45,0 & 45,9 & 45,4 & 53,4 & 56,6 & $55,0^{*}$ \\
\hline $6-13$ & 43,3 & 38,8 & 41,3 & 32,1 & 31,8 & $32,0^{*}$ \\
\hline Total & 100 & 100 & 100 & 100 & 100 & 100 \\
\hline $\begin{array}{l}\text { \% Hombres } \\
\text { * Significat }\end{array}$ & $\begin{array}{l}53,97 \\
5 \%\end{array}$ & & & 50,38 & & \\
\hline
\end{tabular}

Tabla 2

Estado nutritivo por sexo (P/E, OMS ) en 260 niños impedidos

\begin{tabular}{|c|c|c|c|c|c|c|c|}
\hline \multirow[b]{2}{*}{ listado nutritivo } & \multicolumn{2}{|c|}{ Hombres } & \multicolumn{2}{|c|}{ Mujeres } & \multicolumn{3}{|c|}{ Total } \\
\hline & 10 & $\%$ & $\mathrm{~N}^{\circ}$ & $\%$ & No & $\%$ & \\
\hline Obeso & 5 & 3,8 & 2 & 1,6 & 7 & 2,7 & \\
\hline Sotrepeso & 3 & 2,3 & 3 & 2,3 & 6 & 2,4 & \\
\hline Eutrofia & 31 & 23,7 & 31 & 24,0 & 62 & 23,8 & \\
\hline$D_{1}$ & 45 & 34,4 & 43 & 33.3 & 88 & $33,8)$ & \\
\hline $\mathrm{D}_{2}$ & 34 & 25,9 & 33 & 25,6 & 67 & 25,8 & $71 \%$ \\
\hline$D_{3}$ & 13 & 9,9 & 17 & 13,2 & 30 & 11,5 & \\
\hline Total & 131 & 100,0 & 129 & 100,0 & 260 & 100,0 & \\
\hline
\end{tabular}

E] $71 \%$ de los nī̄os presentó algún grado de D, sin variaciones de importancia por sexo. 
Tabla 3

Estado nutritivo (P/1:, OMS), en diferentes gnupos etarjos 260 ninos impedidos

\begin{tabular}{|c|c|c|c|c|c|c|c|c|c|}
\hline \multirow[b]{2}{*}{ Estado nutritivo } & \multicolumn{2}{|c|}{-2 años } & \multicolumn{2}{|c|}{2 a 5} & \multicolumn{2}{|c|}{6 a 14} & \multicolumn{2}{|c|}{ Total } & \\
\hline & No & $\%$ & $\mathrm{~N}^{\circ}$ & $\%$ & $\mathrm{~N}^{\circ}$ & $\overline{\psi_{0}}$ & $N^{o}$ & $\%$ & \\
\hline Obeso & 1 & 2,9 & 6 & 4,2 & 0 & 0 & 7 & 2,7 & \\
\hline Sobrepeso & 2 & 5,9 & 3 & 2,1 & 1 & 1,2 & 6 & 2,4 & \\
\hline Eutrofia & 9 & 26,5 & 38 & 26,6 & 15 & 18,0 & 62 & 23,8 & \\
\hline$D_{1}$ & 14 & 41,2 & $\$ 2$ & 36,4 & 22 & 26,6 & 88 & 33,8 & \\
\hline$D_{2}$ & 6 & 17,6 & 32 & 22,4 & 29 & 34,9 & 67 & 25,8 & $71 \%$ \\
\hline $\mathrm{D}_{3}$ & 2 & 5,9 & 12 & 8,3 & 16 & 19,3 & 30 & 11.5 & \\
\hline Total & 34 & 100,0 & 143 & 100,0 & 83 & 100,0 & 260 & 100,0 & \\
\hline
\end{tabular}

Se observa un aumento en ia prevalencia de D desde lactantes $(64,7 \%)$ a preescolares $(67,1 \%$. y escolares $(80,8 \%)$ Este incremento es. además. en base de los grados avanzados de $D$.

Tabla 4

Estado nlutritivo P/T O.M.S. en diferentes grupos etarios

\begin{tabular}{|c|c|c|c|c|c|c|c|c|c|}
\hline \multirow[b]{2}{*}{ Estado Nutritivo } & \multicolumn{2}{|c|}{ - 2 años } & \multicolumn{2}{|c|}{$2-5$ Años } & \multicolumn{2}{|c|}{$6-14$ Años } & \multicolumn{2}{|c|}{ Total } & \multirow[b]{2}{*}{. } \\
\hline & $N^{0}$ & $\%$ & No & $\%$ & No & $\%$ & No & $\%$ & \\
\hline Obeso & 3 & 8,8 & 9 & 6,3 & 0 & 0 & 12 & 4,6 & \\
\hline Sobrepeso & 3 & 8,8 & 14 & 9,8 & 3 & 3,6 & 20 & 7,7 & \\
\hline Eutrofia & 14 & 41,2 & 60 & 42,0 & 39 & 47,0 & 113 & 43,5 & \\
\hline $\mathrm{D}_{\mathrm{l}}$ & 10 & 29.4 & 36 & 25,1 & 26 & 31,3 & 72 & $27, ?$ & ) \\
\hline $\mathrm{D}_{2}$ & 4 & 11,8 & 19 & 13,3 & 14 & 16,9 & 37 & 14,2 & $44,2 \%$ \\
\hline $\mathrm{D}_{3}$ & 0 & 0,0 & 5 & 3,5 & 1 & 1,2 & 6 & 2,3 & \\
\hline Total & 34 & 100,0 & 143 & 100,0 & 83 & 100,0 & 260 & 100.0 & \\
\hline
\end{tabular}

tró un $47,3 \%$ de frecuencia, cifra similar a la observada en el total de la muestra cstudiada. Los grados $D_{2}$ y $D_{3}$, tampoco fueron significati. vamente superiores entre eștos nin̄os.

\section{DISCUSION}

Lo más relevante de los resultados obtenidos en la elevada prevalencia de desnutrición en la población estudiada.

Las cifras de prevalencia de 1985 , entregadas por el Ministeriv de Salud, son un $8,3 \%$ (promedio nacional) y $8,5 \%$, (Región Metropolitana) en niños menores de 6 años, evaluados con la relación $P / E$ Sempé. ${ }^{5}$ Las cifras nacionales de desnutrición P/T, O.M.S., corresponden a un $3,5 \%$ en el Area Metropolitana.

Sin embargo en el I.R.J., el $4 \%$ de los menores de 6 años presenta algún grado de desnutrición P/T, O.M.S.

Las diferencias observadas entre la relación $\mathrm{P} / \mathrm{E}$ y $\mathrm{P} / \mathrm{T}$ se deben a que un gran número dc niños impedidos presenta un déficit importante en su talla. ya sea por su patología de base o por la desnutrición crónica, presente en muchos de ellos.

En el proceso de desnutrición el parámetro más sensible es el peso: es el primero que se compromete, como asi mismo es el primero que se recupera, cuando el desnutrido mejora. En cambio, la talla se compromete cuando la desnutrición se prolonga por un tiempo mayor. Mientras más antigua es la desnutrición, mayor es el compromiso de la talla.

A] recuperarse un desnutrido crónico se produce un incremento de peso que no se acompaña de un aumento proporcional de talla. Esto se refleja en un mejoramiento de la relación $\mathrm{P} / \mathrm{T}$, llegando ésta incluso a márgenes normales; lo que comúnmente llamamos "enanismo nutricional".

A medida que el niño avanza en edad se agrava la desnutrición $P / T$. La relación $P / T$ nos indica si el niño está o no compensado desde el punto de vista nutricional. Con estas premisas, el $44,2 \%$ de 
los niños del l.R.I. se encuentran nutricionalmente descompensados. Esto significa que, in. cluso considerando el déficit de la talla: presente por la patología de base, casi la mitad de ellos presenta un déficit de peso para la estatura.

La desnutrición pucde ser de iniciación precoz (antes de los 6 meses de vida), intermedia (entre 6 meses y 2 años) o tardia (despućs de dos años). Cuando el comienzo es más precoz. más graves son las secuelas. El pronóstico psiconeurológico depende de la edad de inicio y de la intensidad $y$ duración del déficit nutricional.

Es preoçupante el hecho que el $41 \%$ de los lactantes que ingresa al I.R.I. presenta algún grado de desnutrición $\mathrm{P} / \mathrm{T}$ y que un $64.7 \%$ tenga desnutrición P/E. Extrapolando al total de ingresos podemos decir que en 1985 se recibieron 75 lactantes con desnutrición $\mathrm{P} / \mathrm{E}$ o 44 con desnutrición $\mathrm{P} / \mathrm{T}$.

En este estudio se observa que los escolares y preescolares son los más afectados por la desnutrición tanto en intensidad como en frecuencia. Deseraciadamente, estos niños no son acogidos por los Consultorios Periféricos en planes especiales de recuperación nutricional.

Eśs sabido que cuando el niño no recibe un aportc energético adecuado el crecimiento se produce a expensas de sus propias proteínas, especiamente musculares. Esto conlleva lesiones de su sistema locomotor, las que aguavan su patología de base, prolongan su proceso de rebabilitación y lo hacen más difícil. Por to tanto, es indiscutible que el rendimiento de la rehabilitación depende entre otros factores del estado nutritivo.

Se ha observado que, incluso en niños mayores. la recuperación nutricional mejora los puntajes de pruebas psicológicas (Gesell, Goodenough y otros) ${ }^{6}$

Otro aspecto importante de comentar es el elevado porcentaje de pacientes con parálisis cerebral (60\% de los ingresos). Ellos, en su gran mayoría, dependen de terceras personas para alimentarse. Si a ésto agregamos que el nivel sociveconómico y cultural es, en general, bajo nos enfrentamos a un problema aún más dificil de resolver. De hecho, las madres frecuentemente refieren que administran los alimentos a estos niños, incluso cscolarcs, en mamaderas porque es la forma más fácil de hacerlo. Habitualmente se trata de madres con varios hijos y poca disponibilidad de tiempo que, por lu general, no han recibido orientación y apoyo nutricional para el manejo de sus hijos. Esto significa que un gran número de niños impedidos reciben diariamente aportes calóricos y de nutrientes esenciales muy por debajo de sus requerimientos.

En medios hospitalarios existe preocupación por el deterioro nutritivo que sufre el nin̄o hospitalizado $y$, en consecuencia, se han implementado medidas de apoyo nutricional intensivo (alimentación enteral y parenteral) en los casos más agudos, permitiendo disminuir la morbilidad asociada y acortar el tiempo de recuperación. Esto no ocurre, hasta ahora, en niños impedidos.

$\mathrm{Es}_{s}$ indudable que nuestros pacientes están condicionados por múltiples factores que inciden en el deterioro de su estado nutritivo $y$ que el problema no es fácil de resolver, entre otras cosas, por lo transitorio de su paso por la lnstitución.

Creemos que las cifras obtenidas en este estudio no pucden dejarnos indiferentes, más aún sabiendo que la desnutrición es una condición reversible aút1 en niños impedidos. Nos parece indispensable evaluar periodica y sistemáticamente el estado nutritivo de todo paciente crónico, para asi brindar una atención médica integra].

\section{RESUMEN}

En uria muestra del $30 \%$ (260 casos) de los menores de 15 años, niños ingresados al Instituto de Rehabilitación Infantil durante 1985, se evaluo el estado nutritivo con los valores normales de la O.M.S. mediante la relación peso edad (P/E) y pesu talla (P/T). La prevalencia de desnutrición (D) fue de $71 \%$ con la relạción $P / E$ y $44.2 \%$ con lá relación $\mathrm{P} / \mathrm{T}$. Estas cifras son significativamente más altas que las de la población general, siendo la D grado III un $11,5 \%$ (30 casos) con $P / T$ y un $2,3 \%$ (6 casos) con P/T. La prevalencia de D fue mayor en los grupos de edad escolar (6 a 14 años) llegando entre ellos a 80.8 con la relación $\mathrm{P} / \mathrm{E}$ y a $49,4 \% \mathrm{con} \mathrm{P} / \mathrm{T}$.

\section{REFERENCIAS}

1. Pino, S., Vargas, $\mathcal{N}_{\text {, }}$ Blanco, M.A., Mayne, D. Fstado nutritivo en niños impedidos. Presentado a Instituto de Rehabilitacion Infantil. Mayo 1983.

2. Medición del evento nutricional de programas de suplementación alimentaria a grupos vulnerables WHO/FAP/79. Organización Mundjal de la Salud. Ginebra, 1980.

3. Lewin, L., Vargas, N., Belmar. A., Campero, M.: Sensibilidad de tres criterios para el diagnóstico de desnutrición en lactantes. Rev Chil Pediat 5: 345 , 1986.

4. Sobarzo, 1 , Díaz. E., Krause, $S$.: Fistado nutritivo eл una población escolar rural. Rev Chil Pediatr 55: 59, 1984.

5. Población en control de menores de seis años, según estado de nutrición. Énero 1985-1986. Ministerio de Salud, 1986.

6. Meneghello, Julto: Pediatria. Ed. Intermédicd. Buenos Aires, 2a Ed. 1978, Vol. 1, pág. 292. 